University of Nebraska - Lincoln

DigitalCommons@University of Nebraska - Lincoln

July 1994

\title{
Axial asymmetry in the nuclear magnetic resonance spectra of deuterated methyl groups: An alternative explanation
}

\author{
Mieng-Hua Wann \\ University of Nebraska - Lincoln \\ Gerard S. Harbison \\ University of Nebraska - Lincoln, gharbison1@unl.edu
}

Follow this and additional works at: https://digitalcommons.unl.edu/chemistryharbison

Part of the Chemistry Commons

Wann, Mieng-Hua and Harbison, Gerard S., " Axial asymmetry in the nuclear magnetic resonance spectra of deuterated methyl groups: An alternative explanation " (1994). Gerard Harbison Publications. 2.

https://digitalcommons.unl.edu/chemistryharbison/2

This Article is brought to you for free and open access by the Published Research - Department of Chemistry at DigitalCommons@University of Nebraska - Lincoln. It has been accepted for inclusion in Gerard Harbison Publications by an authorized administrator of DigitalCommons@University of Nebraska - Lincoln. 


\title{
Axial asymmetry in the nuclear magnetic resonance spectra of deuterated methyl groups: An alternative explanation
}

\author{
Mieng-Hua Wann ${ }^{\text {a) }}$ and Gerard S. Harbison ${ }^{\text {b) }}$ \\ Department of Chemistry, University of Nebraska at Lincoln, Lincoln, Nebraska 68588-0304
}

(Received 7 January 1994; accepted 11 February 1994)

\begin{abstract}
The deuterium nuclear magnetic resonance (NMR) powder spectra of methyl groups at ambient temperatures are invariably averaged by rotation or hopping about the $C_{3}$ axis. The canonical result for a perfectly threefold symmetric methyl is an axially symmetric Pake doublet whose splitting, in the case of perfect tetrahedral angles around the group, is averaged by a factor of exactly three. However, deuterium NMR spectra of methyl groups are often somewhat axially asymmetric, indicating a significant deviation either of the methyl group, or of its environment, from threefold symmetry. Previous explanations for this phenomenon have hinged on either an additional motional mechanism along a different axis, or on a perturbation of the field gradient around the methyl group by electronegative groups. We propose a simpler explanation; it may arise in a distortion of the methyl geometry itself, so that the group no longer has perfect rotational symmetry. We analyze some experimental examples of distorted methyls, and compare the extent of distortion required by the NMR data with those observed in published neutron structures.
\end{abstract}

\section{INTRODUCTION}

The changes wrought by motion on deuterium nuclear magnetic resonance (NMR) line shapes give the experimentalist one of the most direct methods of characterizing dynamical processes in the solid state. The deuterium NMR spectrum at high field is dominated by the electric quadrupole coupling, which arises from the interaction between the quadrupole moment of the (spin 1) deuteron on the one hand, and the gradient of the electric field on the other. This interaction is in many cases extraordinarily simple and predictable, for example, the deuterium quadrupole coupling tensors of deuterium involved in C-D bonds in organic molecules are almost invariably axially symmetric and have quadrupole coupling constants $e^{2} q Q / h$ of $167 \pm 10 \mathrm{kHz} .{ }^{1}$ Aromatic deuterons have coupling constants about $10 \%$ larger, and often deviate slightly from perfect axial symmetry about the C-D bonds, but the asymmetry parameter $\eta$ is never more than $0.1 .^{2}$ Even acidic deuterons in C-D bonds such as in malonic acid fall within this narrow range. ${ }^{3}$

The indifference of the organic deuteron to chemistry is a boon to the solid-state spectroscopist, since it allows the spectrum to be treated purely in terms of dynamics. Dynamical influences on the spectrum can be separated into several motional regimes. Slow motions (with time constants greater than $10^{-4} \mathrm{~s}$ ) have no effect on the directly observed deuterium spectrum, and can be detected only by their influence on Zeeman or quadrupolar order. Motions in the so-called intermediate exchange time scale $\left(10^{-4} \mathrm{~s}>\tau_{C}>10^{-6} \mathrm{~s}\right)$ cause spectral changes which depend on the mode of motion (more specifically, the jump-angle for the C-D bond, and the number of exchanging sites), and on the time constant(s) of the dynamics. Faster motions are affected by the mode of motion but not by the rate, which latter, however, can generally be obtained from relaxation measurements.

a) Present address: Sloan-Kettering Cancer Institute, New York, New York.

b) Author to whom correspondence should be addressed.
Unfortunately, deuterium line shapes are seldom unambiguous about the precise mode of motion. Generally some other information, either from NMR or from a knowledge of the symmetry of the system, must be introduced in order to generate molecular information from the line shape. In the special case of axially symmetric motion about a single axis, for example, deuterium NMR line shapes do not distinguish between three-site jumps, six-site jumps, or continuous rotational diffusion. This problem was confronted by Batchelder et $a l^{4,5}$ for the deuterium NMR spectrum of $L$-alanine $\left(2-d_{3}\right)$. They showed the fast limit deuterium line shape is consistent with an axially symmetric hopping motion between $N$ equivalent sites, about a jump axis oriented at an angle of $\theta=110^{\circ}$ with respect to the $\mathrm{C}-\mathrm{D}$ axes, where $N$ can be any integer higher than 3 . Relaxation measurements were used to determine that $N$ was in fact equal to 3 . In this particular case (axially symmetric spectrum resulting from an $N$-site hop) the only information available from the spectrum is the angle $\theta$. This angle is directly related to the motional scaling of the Pake splitting, defined by $f$, the ratio of motionally averaged to unaveraged Pake splittings,

$$
f=\left(3 \cos ^{2} \theta-1\right) / 2 \text {. }
$$

Surprisingly, however, some methyl group spectra at room temperature deviate from pure axial symmetry. Deviations of this sort can be quantified by an asymmetry parameter $\eta$,

$$
\eta=\left(Q_{22}-Q_{11}\right) / Q_{33},
$$

where $Q_{n n}$ are the principal values of the averaged quadrupolar interaction tensor. Asymmetry parameters of as large as $0.07-0.08$ have been observed for thymine- $d_{3}$ (Ref. 6) and for thymidine (Ref. 7). Similar $\eta$ values were noted by Schwartz et al. ${ }^{8}$ in hexamethylbenzene, while values of 0.088 and 0.144 were measured by Barnes ${ }^{9}$ for acetic acid and acetone, respectively.

A variety of explanations have been suggested for this phenomenon. Hiyama et al. ${ }^{6}$ proposed that the effect is elec- 
trostatic in origin. An electronegative group close to the methyl contributes to the electric field gradient in a way that distinguishes between the $x$ and $y$ directions. Barnes ${ }^{9}$ proposed a similar explanation for acetyl groups; he noted that a $10 \%$ difference in rigid-lattice coupling constants between the three averaged sites could explain the observed $\eta$. It is however difficult to apply this reasoning to hexamethylbenzene, a nonpolar molecule. Schwartz et al. ${ }^{8}$ suggested that deuterium-deuterium dipolar interactions could mimic the effect of $\eta$; the size of these interactions is however difficult to reconcile with the magnitude of the observed effect. Kintanar et al. ${ }^{10}$ proposed that the effect in thymidine derives from additional dynamics, such as a wagging motion perpendicular to the methyl symmetry axis. All of these diverse possibilities have in common the feature that they presume some feature extrinsic to the methyl group itself as the source of the perturbation.

\section{THEORY}

In this paper we propose that no external perturbation is in fact necessary; that methyl groups undergoing threefold hops on their own generate moderate asymmetry parameters, if the methyl group has lower than $C_{3}$ symmetry. The effects considered here are ascribed to distortion of the methyl group by a twofold symmetric environment. This assumption will be justified in the Discussion. For now, we will consider the possible distortions of a methyl group which still retain a twofold plane of symmetry. We shall ignore bond-length ef-
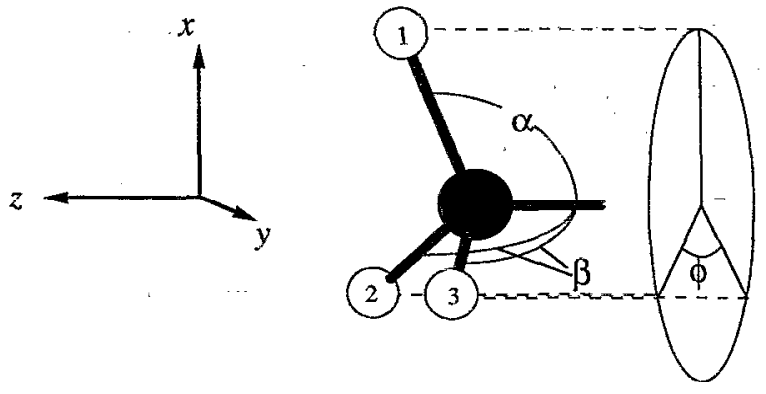

FIG. 1. Methyl group distorted in a twofold symmetric environment, showing the definitions of the angles and coordinate system used in the text.

fects, concentrating only on changes in bond angles. These constraints limit the distortion of the methyl to three degrees of freedom, which we have chosen to represent by three angles $\alpha, \beta$, and $\phi$. These angles are depicted in Fig. 1. In the figure, the plane of symmetry passes vertically through deuteron 1 (D1), the methyl carbon $\mathrm{C}$, and its bond to the rest of the molecule $(\mathrm{R})$. The angle between $\mathrm{C}-\mathrm{D} 1$ and $\mathrm{C}-\mathrm{R}$ is defined as $\alpha$, the angle between $\mathrm{C}-\mathrm{D} 2$ (or $\mathrm{C}-\mathrm{D} 3$ ) and $\mathrm{C}-\mathrm{R}$ is $\beta$. Finally, $\phi$ is the angle of rotation about $\mathrm{C}-\mathrm{R}$ which takes D2 onto D3.

We also define a Cartesian coordinate system in which $z$ is along the $\mathrm{C}-\mathrm{R}$ bond, $x$ is normal to $z$ and within the plane of symmetry, and $y$ is normal to both. These axes are also sketched in Fig. 1. The truncated quadrupolar tensor in frequency units in this coordinate system is

$$
\frac{3 e^{2} g Q}{8 h}\left\{\begin{array}{ccc}
{\left[\cos ^{2} \alpha+(3 \cos 2 \beta-1) \cos ^{2} \psi\right.} & & \\
\left.-2 \sin ^{2} \alpha+2 \sin ^{2} \psi\right] / 3 & 0 & (\sin 2 \alpha) / 2+\cos \psi \sin 2 \beta \\
0 & (1+\cos 2 \beta+\cos 2 \psi & 0 \\
& -\cos 2 \beta \cos 2 \psi) / 2 & \\
(\sin 2 \alpha) / 2+\cos \psi \sin 2 \beta & 0 & -(1+\cos 2 \alpha+2 \cos 2 \beta) / 2
\end{array}\right\}
$$

where $\left(e^{2} q Q / h\right)$ is the rigid-lattice deuterium quadrupole coupling constant, and the angle $\psi=\pi-\phi / 2$. If $\alpha=\beta=\cos ^{-1}(-1 / 3)$ and $\phi=2 \pi / 3$ (as is the case for a tetrahedrally symmetric methyl group) the off-diagonal elements are zero and the diagonal elements become. $\left(e^{2} q Q / h\right)(1 / 8,1 / 8,-1 / 4)$, the canonical values for a methyl group, one third of those of the rigid lattice deuteron.

It is apparent from the above matrix that the $y y$ principal axis will remain along the normal to the plane of symmetry, irrespective of how large the distortion may be. However, the off-diagonal $x z$ elements mean that the $Q_{11}$ and $Q_{33}$ principal axes will be perturbed away from the $x$ and $z$ directions. This matrix can be diagonalized analytically; however, the functional form of the eigenvalues is too lengthy to be given here, and may easily be obtained by the interested investigator.

Defining $f$ as the ratio between the largest motionally averaged principal value and the largest rigid lattice principal value

$$
f=\left|\frac{Q_{33}}{\left(3 e^{2} q Q^{/ 4 h)}\right.}\right|
$$

and with $\eta$ as already defined [Eq. (2)] we can now examine the dependencies of these two measurables on the three distortion angles $\phi, \alpha$, and $\beta$. These dependencies are depicted in Figs. 2-4, respectively.

Examination of the figures shows clearly that, while the asymmetry parameter is strongly dependent on all three angles, the reduced coupling constant $f$ depends primarily on $\alpha$ and $\beta$; the dependence on $\phi$ is second order and insignificant for small perturbations away from the canonical tetrahedral value. The second-order nature of this dependence suggests that perturbation theory may usefully be used to simplify this problem. The quadrupolar tensor contains only 

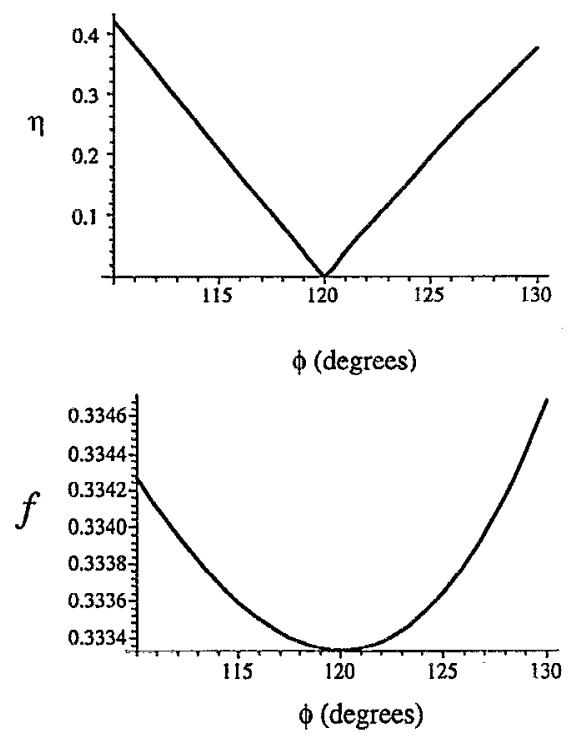

FIG. 2. Dependence of the quadrupolar asymmetry parameter $\eta$, and the dynamical scaling of the quadrupole coupling constant $f$, on the value of the angle $\phi$, defined in the text and in Fig. 1. Note the marked dependence of $\eta$ on $\phi$, vividly contrasting with the virtual independence of $f$.

one pair of off-diagonal elements $Q_{13}=Q_{31}$, and these connect diagonal values which, for methyl geometries close to tetrahedral, are very different; for a perfect tetrahedron $Q_{33}$ is the unique principal element and $Q_{11}=-Q_{33} / 2$. For geometries close to tetrahedral, to first order in perturbation theory

$$
\begin{aligned}
& f \cong-(1+\cos 2 \alpha+2 \cos 2 \beta) / 4, \\
& \eta \cong \frac{-1-f+(1-\cos 2 \beta)(1-\cos \phi) / 2}{f} .
\end{aligned}
$$
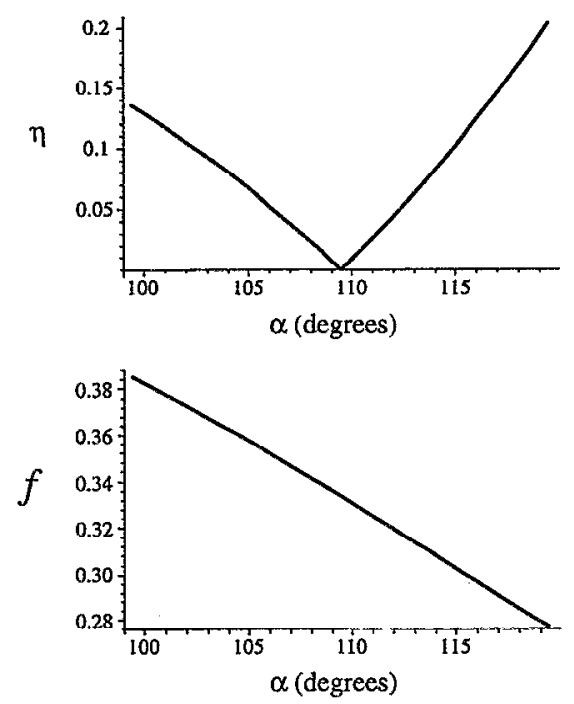

FIG. 3. Dependence of $\eta$ and $f$ on the value of the angle $\alpha$, defined in the text.
These expressions are accurate to $\pm 1 \%$ for values of $\alpha$ and $\beta$ within $\pm 7^{\circ}$ and for $\phi$ within $\pm 5^{\circ}$ of their tetrahedral values. This range spans all of the experimental data reported by us and others for methyl group asymmetry parameters.

\section{METHODS}

\section{A. Synthesis of octatrienylideneimines}

Crotonaldehyde (2-butenal) was obtained from Aldrich Chemicals and was dried by azeotropic distillation at ambient pressure. $50 \mathrm{~g}$ of the dried material was distilled at room temperature and 5 Torr pressure and then deaerated with nitrogen. $\mathrm{D}_{2} \mathrm{O}(100 \mathrm{~g})$ and $3 \mathrm{~m} \ell$ of concentrated $\mathrm{DCl}$ in $\mathrm{D}_{2} \mathrm{O}$ (both Cambridge Isotopes) were added. After refluxing for $36 \mathrm{~h}$ under nitrogen, the reaction mixture was cooled with an ice bath, neutralized with a saturated solution of $\mathrm{NaHCO}_{3}$ in water, diluted with $200 \mathrm{~m} \ell$ ether, and the organic layer was separated. The aqueous layer was washed several times with ether, and the washings were combined with the organic layer. After drying over anhydrous $\mathrm{Na}_{2} \mathrm{SO}_{4}$, the ether was removed by rotary evaporation. Most of the unreacted labeled crotonaldehyde was collected by simple distillation. The remaining dark brown oil was distilled under vacuum and gave four distinct fractions. The first fraction (room temperature, 10 Torr) contained unreacted but partially exchanged crotonaldehyde. The second fraction $\left(65^{\circ} \mathrm{C}, 10\right.$ Torr) contained a cyclic side product, 6-methyl-1,3cyclohexadiene-1-carboxaldehyde; the extent of deuteration of this product was not ascertained. Fraction $3\left(65-72{ }^{\circ} \mathrm{C}\right.$, 0.2 Torr) was $\left[1,1,1,3,5,7_{-}{ }^{2} \mathrm{H}_{6}\right]-2,4,6$-octatrienal, which solidified in the distillation head. Crystallization of this fraction from $n$-hexane gave a faintly yellow powder which melted at $56-57^{\circ} \mathrm{C}$. ${ }^{1} \mathrm{H}$ NMR in solution in $\mathrm{CDCl}_{3}$ showed it to be approximately $35 \%$ deuterated at the $1,3,5$, and 7 positions. The fourth fraction contained $\left[1,1,1,3,5,7,9,11-{ }^{2} \mathrm{H}_{8}\right]$
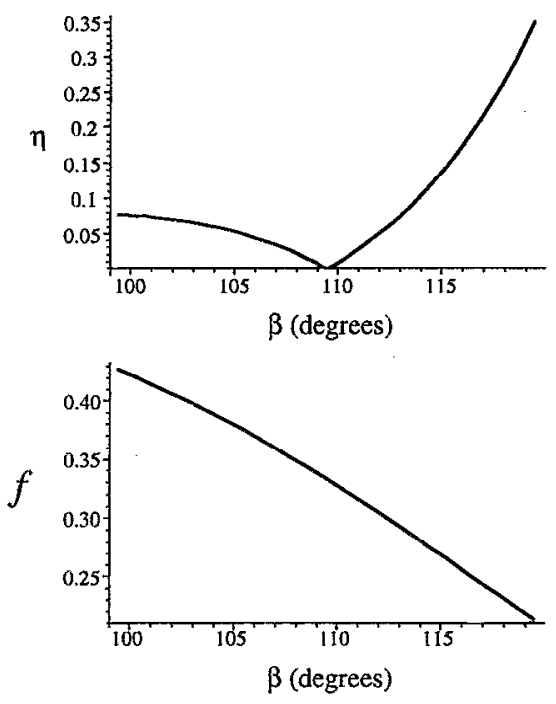

FIG. 4. Dependence of $\eta$ and $f$ on the value of the angle $\beta$, defined in the text. 
-2,4,6,8,10-dodecapentaenal which crystallized from an $80 / 20$ methanol-water mixture to yield an orange-yellow powder with a melting point of $166-167^{\circ} \mathrm{C}$.

The Schiff bases $\left[1,1,1,3,5,7-{ }^{2} \mathrm{H}_{6}\right] N$-butyl $2,4,6$ octatrienylideneimine (BOTI) and $\left[1,1,1,3,5,7-{ }^{2} \mathrm{H}_{6}\right] N$-pentyl 2,4,6-octatrienylideneimine (POTI) were formed by combining equimolar quantities of the $\left[1,1,1,3,5,7-{ }^{2} \mathrm{H}_{6}\right]$ $-2,4,6$-octatrienal with $n$-butylamine and $n$-pentylamine, rcspectively, in acetonitrile at room temperature. They precipitated from solution within 5 min. After drying in vacuo, the products were vacuum-sublimed at 0.1 Torr, then characterized by solution NMR spectroscopy. The crystalline products were white to off-white platelets, which tend to discolor on exposure to light and air, but were stable under nitrogen at $-20^{\circ} \mathrm{C}$.

\section{B. NMR spectroscopy}

Most ${ }^{2} \mathrm{H}$ NMR spectra were obtained at a frequency. of $61.42 \mathrm{MHz}$ using a Bruker MSL-400 spectrometer. A quadrupole echo pulse sequence was typically employed, with a delay $\tau_{E}=12 . \mu$ s between the two pulses of the quadrupole echo. The $\pi / 2$ pulse was $5 \mu \mathrm{s}$ for BOTI and $3.2 \mu \mathrm{s}$ for POTI. These pulse lengths would be inadequate for work on rigidlattice deuterium line shapes, but are adequate for methyl group spectra in the fast motional regime. Dwell times were $4 \mu \mathrm{s}$, corresponding to a spectral width of $\pm 125 \mathrm{kHz}$. Recycle delays ranged from $3 \mathrm{~s}$ at room temperature to $640 \mathrm{~ms}$ at low temperature.

To study the effect of spin-lattice relaxation, ${ }^{2} \mathrm{H}$ NMR line shapes were obtained as a function of a variable interval $\tau_{R}$ using a three-pulse inversion recovery sequence,

$$
\pi-\tau_{R}-\pi / 2-\tau_{E}-\pi / 2 \text { - acquire. }
$$

Magnetization recovery was measurcd scparately for the parallel and perpendicular edges of the Pake doublet, and was fit using an iterative linear least squares program.

Proton-decoupled deuterium spectra were obtained at a field of 7.1 T, using a double-tuned probe. ${ }^{11}$ A quadrupole echo sequence was used, with deuterium $\pi / 2$ pulse lengths of $2.8 \mu \mathrm{s}$ and a $\tau_{E}$ delay of $20 \mu \mathrm{s}$. The proton-decoupling of field corresponded to a frequency of $85 \mathrm{kHz}$.

\section{RESULTS}

A room tempcrature, proton decoupled spectrum of BOTI is shown in Fig. 4. It comprises a single, axially asymmetric Pake doublet which is obviously motionally averaged; we assign the spectrum to the labeled methyl deuterons. There is no evidence for the deuterons at the 3,5 , and 7 positions. We attribute this absence to saturation of these signals at the $3 \mathrm{~s}$ repetition time. Presumably, therefore their $T_{1} \mathrm{~s}$ are much greater than $3 \mathrm{~s}$, which is not unusual for deuterons attached to what is probably a rigid conjugated polyene chain. The same compound labeled at the $1^{\prime}$ position shows a rigid-lattice Pake doublet and a long $T_{1}(12)$. The center spike is due to isotropic impurities. The spectrum was fit to a Pake doublet with a parallel-edge frequency $Q_{33}$ of 39 $\mathrm{kHz}$ and an asymmetry parameter $\eta$ of 0.13 .

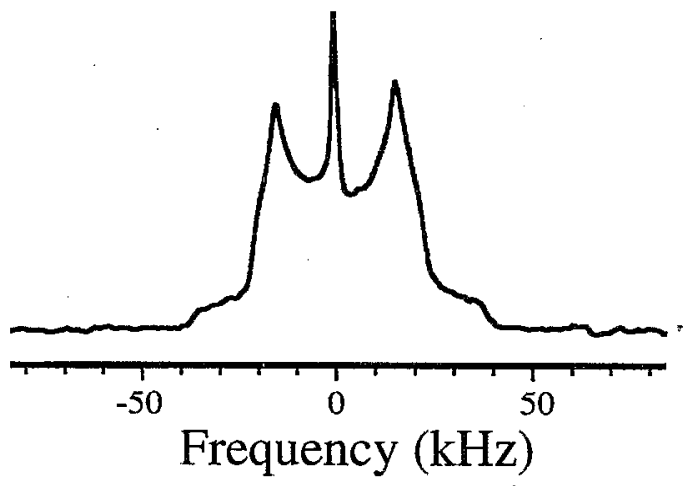

FIG. 5. Room temperature proton-decoupled deuterium NMR spectrum of $\left[1,1,1,3,5,7-{ }^{2} \mathrm{H}_{6}\right] N$-butyl $2,4,6$-octatrienylideneimine. Owing to the rapid repetition, only the methyl spectrum is seen; the comparatively large asymmetry parameter is evident as shoulders on the perpendicular edge of the spectrum.

It is notable that the sharpness of the shoulders is dependent on proton decoupling, without decoupling, these features are considerably smeared out by dipolar interactions with nearby protons. Broadening by dipolar coupling to protons is not usually considered to be a hindrance in deuterium NMR. However in this case the low isotopic enrichment means that each deuteron likely has one or two accompanying protons" on the methyl, at a distance of 1.6-1.7 $\AA$. Averaged by a factor of 2 by the methyl group hops, this yields a proton deuteron two-spin coupling of $4 \mathrm{kHz}$. Mutual spinflips within the proton spin reservoir will cause this interaction to appear as homogeneous broadening; the effect of this broadening can be appreciated by comparing Fig. 4 with the inversion recovery spectra in Fig. 5, where the singularities are much less distinct.

Inversion recovery spectra were obtained for both BOTI and POTI at a range of temperatures between $135 \mathrm{~K}$ and the temperature at which they transform into a smectic liquidcrystalline phase. Representative spectra for BOTI are shown in Fig. 5. Two major features are apparent. First, the deuterium relaxation time becomes shorter as the temperature is decreased, implying that the methyl group motion leading to the relaxation is fast compared with the Larmor frequency. Second, the relaxation is significantly anisotropic. Examination in particular of the slices near the relaxation null show that the parallel edges of the Pake doublet relax considerably faster than the perpendicular edges. For this reason, the parallel and perpendicular edges were fitted separately to a exponential decay at each temperature for both BOTI and POTI. Average values of the relaxation anisotropy, $T_{1 \text {,parallel }} / T_{\text {1.perpendicular }}$, between 125 and $230 \mathrm{~K}$, were 1.69 for BOTI and 1.75 for POTI. These values imply a three-site jump process rather than a six-site model or continuous rotational diffusion (see below). With the plausible assumption that the quadrupolar interaction dominates spin relaxation, expressions derived by Torchia and Szabo ${ }^{13}$ were used to generate correlation times $\tau_{c}$ for BOTI and POTI at each measured temperature. As can be seen from Fig. 6, the temperature dependence of $\tau_{c}$ follows an Arrhenius relationship closely. The figure shows plots for $\tau_{c}$ derived from perpen- 
(a)

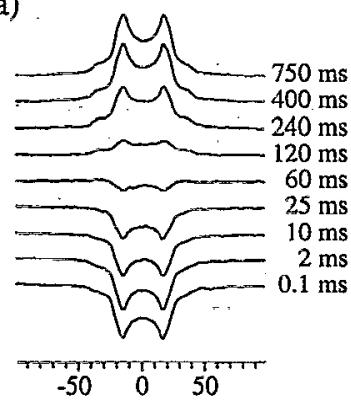

Frequency $(\mathrm{kHz})$ (b)

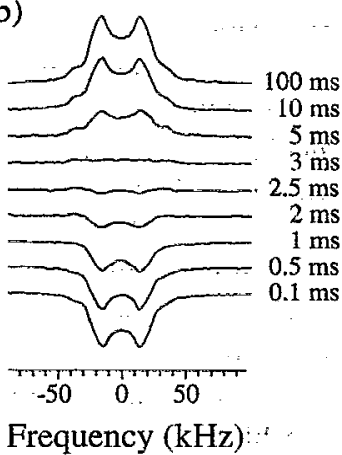

FIG. 6. Inversion recovery deuterium NMR spectra of $\left[1,1,1,3,5,7-{ }^{2} \mathrm{H}_{6}\right] \mathrm{N}$-butyl-2,4,6-octatrienylideneimine, taken at (a) $195 \mathrm{~K}$; (b) $135 \mathrm{~K}$, taken with recovery delays $\tau_{R}$ as shown.

dicular edge data only; parallel edge data gave very similar fits but had a considerably larger scatter, presumably because of the poorer signal to noise. Fits to the Arrhenius equation

$$
\tau_{c}=A_{0} \exp (-k T / \Delta E)
$$

gave the activation energies and pre-exponential factors summarized in Table I.

\section{DISCUSSION}

The fast-limit $T_{1}$ anisotropies in BOTI and POTI are somewhat smaller than the factor of 2.0 expected for a "pure" three-site hop, but they are close to the values measured experimentally for moderate and high-barrier three-site systems. The discrepancy between theory and experiment probably occurs because the methyl group inhabits a threedimensional torsional potential well with a significant width, rather than a threefold delta function potential. The anisotropies clearly distinguish between a sixfold well, as has been observed in certain low-barrier systems such as nitromethane, and rotational diffusion, which might be observed, for example, in a methyl halide in a glassy matrix. The barrier heights in BOTI and POTI are very similar, and close to those measured for a wide range of methyl groups, ranging from alanine derivatives to hexamethylbenzene. The pre-exponential factors are likewise not noteworthy.

The linearity of the Arrhenius plot (Fig. 7) not only sup-ports a discrete $N$-site jump model for the motion, but it also suggests that only a single relaxation mechanism is effective over the range studied. This is of some importance, since, in principle, the asymmetry parameters measured could be caused by an additional motion of the methyl group; either a

TABLE I. Pre-exponential factors, activation energies, and $T_{1}$ anisotropies for the methyl group three-site hops in the two compounds studied in this work.

\begin{tabular}{cccc}
\hline & & Activation energy \\
Compound & $A_{0}(\mathrm{~s})$ & $(\mathrm{kJ} / \mathrm{mol})$ & $T_{1}$ anisotropy \\
\hline BOTI & $10^{-12.7252}$ & 9.12 & 1.69 \\
POTI & $10^{-12.8782}$ & 10.25 & 1.75 \\
\hline
\end{tabular}

librational motion along one axis, or a discrete jump between two different positions of the methyl group. Librational motion is almost ruled out by the fact that $\eta$ is independent of temperature for both compounds. Were the methyl wagging from side to side in a dish-shaped potential minimum, the amplitude of the wagging motion would decrease with temperature, with a corresponding decrease in $\eta$. A two-site hop would give an asymmetry parameter independent of temperature over the range where the hop is fast-limit. Such a motional mechanism is difficult to reconcile with the chemical structure. Additional motion of the methyl group requires torsion about one of the single bonds within the polyene chain, and both intuition and experimental evidence suggest that polyene chain is rigid in these molecules, at least below the phase transition to the smectic phase.

Having discounted other mechanisms as a likely cause of the asymmetry parameter, we must then examine whether our mechanism can account for it. It should be clear immediately that, even after we have restricted ourselves to considering only twofold symmetric distortions of the methyl, the experimental data do not give a unique solution; since there are three degrees of freedom and only two measurables. We will therefore inevitably obtain a set of solutions all satisfying the experimental criteria. For BOTI these solutions are graphed in Fig. 8. One is inclined to favor solutions which entail the minimum overall distortion around the carbon; these are found for values of $\phi$ of about $3^{\circ}$ greater or less than $120^{\circ}$; one such set is $\alpha=\beta=110^{\circ}, \phi=123^{\circ}$. This corresponds to a maximum deviation of less than $2^{\circ}$ for each deuteron from its canonical position. The magnitude of the effect occasioned by such a $2^{\circ}$ distortion may be surprising at first glance, but it should be recalled that the asymmetry parameter is calculated for a quadrupolar tensor already scaled to one third of its full value by dynamics. Thus, the distortions have a proportionately larger effect than they
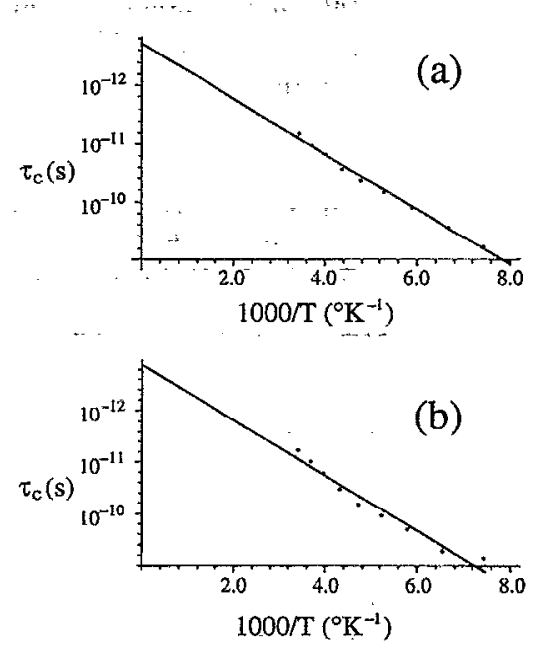

FIG. 7. Arrhenius plot for (a) $\left[1,1,1,3,5,7-{ }^{2} \mathrm{H}_{6}\right] N$-butyl 2,4,6-octatrienylideneimine and (b) $\left[1,1,1,3,5,7-{ }^{2} \mathrm{H}_{6}\right] N$-pentyl $2,4,6$-octatrienylideneimine, plotted together with the best linear least squares fit. 


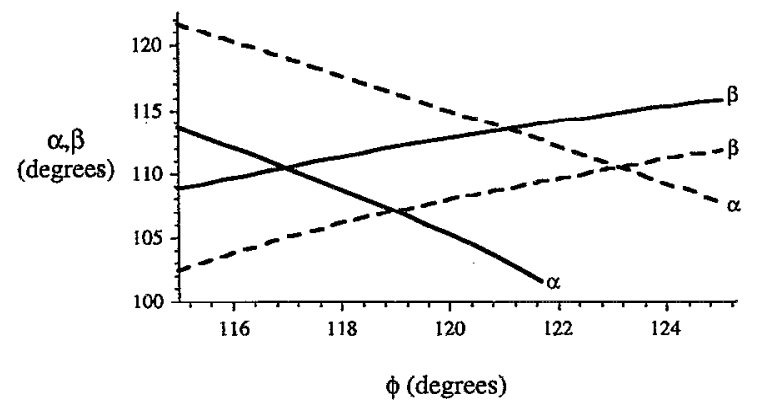

FIG. 8. Values of the angles $\alpha, \beta$, and $\phi$ which fit the measured values of $\eta$ and $f$ for BOTI. The solid line is the fit with $\left|Q_{x x}\right|>\left|Q_{y y}\right|$, the dashed line that with $\left|Q_{y y}\right|>\left|Q_{x x}\right|$.

would for geometries where the dynamical scaling was less severe.

Is a distortion of this magnitude consistent with what is known about the structure of the methyl group? Only in the case of acetic acid can the deuterium spectrum of an asymmetric methyl be directly referenced to a neutron-diffraction structure (which can locate the protons with sufficient accuracy) Using the coordinates given by Jöhnsson, ${ }^{14}$ we calculate the values of $f$ and $\eta$ given in Table II. The methyl group in this compound is moderately distorted in an approximately twofold fashion; the $\mathrm{CCH}$ bond angles are $112.3^{\circ}$, $107.7^{\circ}$, and $108.9^{\circ}$, while the $\mathrm{HCH}$ angles are $111.3^{\circ}, 108.6^{\circ}$, and $108.1^{\circ}$. The calculated asymmetry parameter agrees within $10 \%$ with the measured result of $0.088,{ }^{9}$ showing clearly that in this case that the averaged quadrupolar tensor elements can be explained by distortions of tetrahedral geometry alone.

Unfortunately neutron data are not available for BOTI or POTI, or for that matter for thymidine or hexamethylbenzene. There are, however, published structures for two thymine derivatives, 1-methylthymine (Ref. 15) and 1-methylthymine co-crystallized with 9-methyladenine. ${ }^{16}$ In the latter crystal, the methyl lies on a twofold plane of symmetry, and so fits with our model exactly. From the crystal structure, values of $(\alpha, \beta, \phi)=\left(111.2^{\circ}, 111.2^{\circ}, 116.0^{\circ}\right)$ were obtained, very similar to those estimated for BOTI and
POTI. Using the analytical eigenvalues or the perturbation expression [Eq. (6)] we calculate an $\eta$ of 0.17 , larger than that observed for thymine itself, and slightly in excess of the values obtained for the Schiff bases and for acetone. Similar calculations for 1-methyl thymine lead to best-fit values of $(\alpha, \beta, \phi)=\left(111.5^{\circ}, 111.5^{\circ}, 117.1^{\circ}\right)$ and a smaller asymmetry parameter (0.14).

In both of these neutron structures there is some apparent disorder, and in addition the thermal ellipsoids for the methyl protons are quite large; since thermal averaging may considerably modify quadrupolar coupling constants, it is probably advisable not to place too much reliance on the rather large calculated values of these asymmetry parameters. Nonetheless the data show that using reasonable neutron structural data, $\eta$ values as large or larger than those which have been measured experimentally can be explained solely on the basis of methyl group distortion. It is also instructive to note that the neutron structures of methyls which give axially symmetric spectra, such as $L$-alanine (Refs. $4,17)$ are almost undistorted. In $L$-alanine, the methyl is almost exactly tetrahedral, $(\alpha, \beta, \phi)=\left(110.4^{\circ}, 110.4^{\circ}, 120.8^{\circ}\right)$ and calculated values of averaged quadrupolar parameters $(f=0.311, \eta=0.02)$ agree closely with those measured by previous workers.

We note in closing that in all of these cases where $\eta$ is large, the methyl group is in an environment of approximate twofold symmetry; either a substituent on a planar ring, or attached to an $s p_{2}$ hybridized carbon. It seems that such environments, where the symmetry of the group is incommensurate with that of its environment, lead to the largest distortions of tetrahedral geometry.

\section{CONCLUSIONS}

While this work does not prove that all methyl group asymmetry parameters can be ascribed to deviation of the group from perfect tetrahedral geometry, it shows that even the largest parameters until now observed can be explained fully by such structural distortions, and that where both NMR and accurate structural data are available, there is a close correspondence between calculated and experimental

TABLE II. Measured and/or calculated asymmetry parameters and dynamical scaling factors $f$ (defined in the text) for the compounds discussed in this paper.

\begin{tabular}{llcccc}
\hline \multicolumn{1}{c}{ Compound } & $\eta$ (exp.) & $\eta$ (calc.) $^{\prime}$ & $f$ (exp.) $^{\mathrm{a}}$ & $f$ (calc.) $^{\text {Reference }}$ \\
\hline acetic acid & 0.088 & 0.096 & 0.337 & 0.331 & 9 \\
acetone & 0.144 & $\ldots$ & 0.332 & $\ldots$ & 9 \\
hexamethylbenzene & $0.085^{\mathrm{b}}$ & $\ldots$ & 0.311 & $\ldots$ & 8 \\
thymine & 0.075 & $\ldots$ & 0.311 & $\ldots$ & 6 \\
thymidine & 0.06 & $\ldots$ & 0.302 & $\ldots$ & 7 \\
BOTI & 0.13 & $\ldots$ & 0.311 & $\ldots$ & this work \\
POTI & 0.14 & $\ldots$ & 0.309 & $\ldots$ & this work \\
1-methylthymine & $\ldots$ & 0.128 & $\ldots$ & 0.299 & this work \\
1-methylthymine/9-methyladenine & $\ldots$ & 0.177 & $\ldots$ & 0.304 & this work \\
$L$-alanine & 0 & 0.019 & 0.311 & 0.318 & 5, this work \\
\hline \hline
\end{tabular}

${ }^{a}$ A static quadrupole coupling constant of $167 \mathrm{kHz}$ was used in all cases to calculate $f$. In the few examples where this constant was actually determined, it fell within $5 \mathrm{kHz}$ of this value.

bestimated from a figure in the published reference. 
quadrupolar parameters. The methyl asymmetry parameter may well be among the most sensitive probes of such distortions in aliphatic molecules.

Note added in proof. A similar hypothesis has been suggested by G. L. Hoatson, R. L. Vold, and T. Y. Tse, J. Chem. Phys. 100, 4756 (1994).

\section{ACKNOWLEDGMENTS}

This research was supported by grants from the NIH (GM-39071) and by a NSF Presidential Young Investigator Award (DOB 9057765), and by a Camille and Henry Dreyfus Teacher Scholar Award (to G.S.H.). We thank Robert Vold for useful discussions.

${ }^{1}$ M. Rinné and J. Depireux, Adv. Nucl. Quad. Reson. 1, 357 (1974).

${ }^{2}$ R. G. Barnes and J. W. Bloom, J. Chem. Phys. 57, 3082 (1972).

${ }^{3}$ W. Derbyshire, T. C. Gorvin, and D. Warner, Mol. Phys. 17, 401 (1969).

${ }^{4}$ L. S. Batchelder, C. E. Sullivan, L. W. Jelinski, and D. A. Torchia, Proc. Natl. Acad. Sci. U.S.A. 79, 386 (1982).
${ }^{5}$ L. S. Batchelder, C. H. Niu, and D. A. Torchia, J. Am. Chem. Suc. 105, 2228 (1983).

${ }^{6}$ Y. Hiyama, S. Roy, K. Guo, L. G. Butler, and D. A. Torchia, J. Am. Chem. Soc. 109, 2525 (1987).

${ }^{7}$ Y. Hiyama, S. Roy, J. S. Cohen, and D. A. Torchia, J. Am. Chem. Soc. 111, 8609 (1989).

${ }^{8}$ L. J. Schwartz, E. Meirovitch, J. A. Ripmeester, and J. H. Freed, J. Phys. Chem. 87, 4453 (1983).

${ }^{9}$ R. G. Barnes, Adv. Nucl. Quad. Reson. 1, 335 (1974).

${ }^{10}$ A. Kintanar, T. M. Alam, W. C. Huang, C. Schindele, D. E. Wemmer, and G. Drobny, J. Am. Chem. Soc. 110, 6367 (1988).

${ }^{11}$ P. Tang, W.-J. Chien, and G. S. Harbison, Solid-State Nucl. Magn. Reson. 2, 343 (1993).

${ }^{12}$ M.-H. Wann and G. S. Harbison, J. Am. Chem. Soc. 111, 7273 (1974).

${ }^{13}$ D. A. Torchia and A. Szabo, J. Magn. Reson. 49, 107 (1982).

${ }^{14}$ P.-G. Jöhnsson, Acta Crystallogr. B 27, 893 (1971).

${ }^{15}$ A. Kvick, T. F. Koetzle, and R. Thomas, J. Chem. Phys. 61, 2711 (1974).

${ }^{16}$ M. N. Frey, T. F. Koetzle, M. S. Lehmann, and W. C. Hamilton, J. Chem. Phys. 59, 915 (1973).

${ }^{17}$ K. Beshah, E. T. Olejniczak, and R. G. Griffin, J. Chem. Phys. 86, 4730 (1987). 